\title{
Article \\ New Ostrowski-Type Fractional Integral Inequalities via Generalized Exponential-Type Convex Functions and Applications
}

\author{
Soubhagya Kumar Sahoo $^{1}$, Muhammad Tariq ${ }^{2}$ (D), Hijaz Ahmad ${ }^{3}$ (D), Jamshed Nasir ${ }^{4}$, Hassen Aydi 5,6,7,*(D) \\ and Aiman Mukheimer ${ }^{8}$ (D)
}

1 Department of Mathematics, Institute of Technical Education and Research, Siksha O Anusandhan University, Bhubaneswar 751030, India; soubhagyakumarsahoo@soa.ac.in

2 Department of Basic Sciences and Related Studies, Mehran University of Engineering and Technology, Jamshoro 76062, Pakistan; captaintariq2187@gmail.com

3 Section of Mathematics, International Telematic University Uninettuno, Corso Vittorio Emanuele II 39, 00186 Roma, Italy; hijaz555@gmail.com

4 Department of Mathematics \& Statistics, Virtual University of Pakistan, Lahore Campus 54000, Pakistan; jnasir143@gmail.com

5 Institut Supérieur d'Informatique et des Techniques de Communication, Université de Sousse, Hammam Sousse 4000, Tunisia

6 Department of Mathematics and Applied Mathematics, Sefako Makgatho Health Sciences University, Ga-Rankuwa P.O. Box 60, South Africa

7 China Medical University Hospital, China Medical University, Taichung 40402, Taiwan

8 Department of Mathematics and General Sciences, Prince Sultan University, P. O. Box 66833, Riyadh 11586, Saudi Arabia; mukheimer@psu.edu.sa

Citation: Sahoo, S.K.; Tariq, M.; Ahmad, H.; Nasir, J.; Aydi, H.;

Mukheimer, A. New Ostrowski-Type Fractional Integral Inequalities via Generalized Exponential-Type Convex Functions and Applications. Symmetry 2021, 13, 1429. https:// doi.org/10.3390/sym13081429

Academic Editor: Nicusor Minculete

Received: 8 July 2021

Accepted: 29 July 2021

Published: 4 August 2021

Publisher's Note: MDPI stays neutral with regard to jurisdictional claims in published maps and institutional affiliations.

Copyright: (c) 2021 by the authors. Licensee MDPI, Basel, Switzerland. This article is an open access article distributed under the terms and conditions of the Creative Commons Attribution (CC BY) license (https:// creativecommons.org/licenses/by/ $4.0 /)$.

* Correspondence: hassen.aydi@isima.rnu.tn

\begin{abstract}
Recently, fractional calculus has been the center of attraction for researchers in mathematical sciences because of its basic definitions, properties and applications in tackling real-life problems. The main purpose of this article is to present some fractional integral inequalities of Ostrowski type for a new class of convex mapping. Specifically, $n$-polynomial exponentially $s$-convex via fractional operator are established. Additionally, we present a new Hermite-Hadamard fractional integral inequality. Some special cases of the results are discussed as well. Due to the nature of convexity theory, there exists a strong relationship between convexity and symmetry. When working on either of the concepts, it can be applied to the other one as well. Integral inequalities concerned with convexity have a lot of applications in various fields of mathematics in which symmetry has a great part to play. Finally, in applications, some new limits for special means of positive real numbers and midpoint formula are given. These new outcomes yield a few generalizations of the earlier outcomes already published in the literature.
\end{abstract}

Keywords: Ostrowski inequality; Hölder's inequality; power mean integral inequality; $n$-polynomial exponentially s-convex function

\section{Introduction}

The theory of inequalities along with convexity property plays an essential part in present-day mathematical investigation. Numerical analysis relies on numerous mathematical inequalities such as the Simpson inequality, Hermite-Hadamard inequality, Bullen-type inequality, Ostrowski inequality, etc. Recently, a broad exploration has been completed on acquiring different variants of traditional inequalities using different methodologies. An exceptionally intriguing methodology is to obtain a fractional version of the inequalities. Inequalities associated with various forms of fractional operator such as Riemann-Liouville fractional operator, Conformable, Katugampola fractional operator, Tempered fractional 
operator, Generalized proportional fractional, Weighted fractional operator, Caputo fractional operator, etc. assume a critical part in the foundation of the unique solution for fractional differential equations. For some recent articles on fractional inequalities, see References [1-8].

The concept of convexity plays a strong role in the field of mathematical inequalities and mathematical analysis due to the beautiful nature of its classical definition and its algebraic properties. Recently, many authors have explored the close relationship and interrelated work on convexity and symmetry. They have also explained that due to the strong relationship between them, while working on any one of the concepts it can be applied to the other one as well. Convexity theory has gained a lot of attention in recent years and many generalizations and refinements of convexity analysis for inequalities have been found (see, for example [9-15]).

Fractional operator examines the integrals and derivatives of any order $\alpha>0$ be it real or complex valued. It was introduced by Leibniz and Marquis de 1'Hospital in 1695 by talking about the differentiation of functions. In any case, it encountered a fast development throughout the limited time.

In the theory of integral inequalities, Sarikaya et al. [6] introduced the application of fractional integral operator by establishing the fractional analogues of classical Hermite-Hadamard's inequality using convexity. In [16], Dragomir for the first time established fractional versions of Ostrowski-type inequalities.

In 1938, Ostrowski introduced the following useful and interesting integral inequality, (see [17], page: 468).

Let $\varphi: J \subseteq \mathbb{R} \rightarrow \mathbb{R}$ be a differentiable mapping on $J^{o}$, the interior of the interval $J$, such that $\varphi \in \mathcal{L}\left[\eta_{1}, \eta_{2}\right]$, where $\eta_{1}, \eta_{2} \in J$ with $\eta_{2}>\eta_{1}$. If $\left|\varphi^{\prime}(z)\right| \leq K$, for all $z \in\left[\eta_{1}, \eta_{2}\right]$, then the following inequality holds:

$$
\left|\varphi(z)-\frac{1}{\eta_{2}-\eta_{1}} \int_{\eta_{1}}^{\eta_{2}} \varphi(\chi) d \chi\right| \leq K\left(\eta_{2}-\eta_{1}\right)\left[\frac{1}{4}+\frac{\left(z-\frac{\eta_{1}+\eta_{2}}{2}\right)^{2}}{\left(\eta_{2}-\eta_{1}\right)^{2}}\right]
$$

holds, where $K$ is the Lipschitz constant which is equal to sup $\left\{\left|\frac{\varphi(\chi)-\varphi(\phi)}{\chi-\phi}\right| ; \chi \neq \phi\right\}$. The above result (1) is famously known as the Ostrowski inequality. For recent results and related generalizations, variants and extensions about the Ostrowski inequality (see [18-23]). This inequality gives us an upper bound for the approximation of the integral average $\frac{1}{\eta_{2}-\eta_{1}} \int_{\eta_{1}}^{\eta_{2}} \varphi(\chi) d \chi$ by the value of $\varphi(u)$ at the point $\chi \in\left[\eta_{1}, \eta_{2}\right]$.

The Ostrowski inequality has a great number of applications in different fields of mathematical analysis such as numerical analysis and especially in the theory of approximations. This type of analytic inequality and especially the techniques used in this article have applications in various fields in which symmetry plays a significant role.

In the wake of contemplating writing about convexity theory, propelled and motivated by the continuous generalizations and exploration in this interesting field, we discovered that there exists an exceptional class of convexity known as exponential convexity and recently a great number of researchers are working on this concept for its enhancement. Antczak [24] and Dragomir [25] presented the class of exponential-type convexity. Consequently, Awan [26] contemplated and examined another class of exponential convex function. Very recently, Mahir Kadakal and İscan [27] presented another meaning of exponential-type convexity. Studying the above-mentioned papers, we have introduced a new definition in this aspect which is called $n$-polynomial exponential $s$-convex function. Applying this new definition, we have presented our main results as refinements of the Ostrowski inequality. This is the novelty of our proposed work.

The objective of this paper is to obtain some new novel refinements of Ostrowski's inequality basically using $n$-polynomial exponential $s$-convex function for fractional calculus. We initially attain a new fractional version of the Hermite-Hadamard inequality 
using a new class of convexity, namely $n$-polynomial exponential s-convex function. We additionally investigate some important special cases that can be concluded from the presented results of the article. In Sections 6 and 7, we present a few applications of the presented results. We trust that the thoughts and strategies introduced in this paper will rouse intrigued researchers for further research.

Recently, it has been seen that many scientists are interested in big data analysis, deep learning and information theory using the concept of exponentially convex functions. Hence, we believe that the concept of $n$-polynomial exponentially convex function using fractional operator can attract the interest of such scientists for further development in the field of deep learning, data analysis and information theory.

Motivated by the advancement of the theory of fractional calculus, ongoing research and literature about integral inequality and convexity, the present paper is structured in the following way: First, in Section 2, we will give some necessary known definitions and literature. Second, in Section 3, we will explore the concept of $n$-polynomial exponentially $s$-convex function. In addition, algebraic properties and examples for the newly introduced definition are elaborated. In Section 4, we attain a new fractional version of the Hermite-Hadamard-type inequality. Furthermore, in Section 5, we investigate some novel refinements of the Ostrowski-type inequality and some special cases via the Riemann-Liouville fractional integral operator. Finally, in the next Section, we present some applications to special means and midpoint formula.

\section{Preliminaries}

In this Section, we recall some known concepts.

Definition 1 ([28]). Let $\varphi: I \subseteq \mathbb{R} \rightarrow \mathbb{R}$ be a real-valued function. A function $\varphi$ is said to be convex, if

$$
\varphi\left(\chi \eta_{1}+(1-\chi) \eta_{2}\right) \leq \chi \varphi\left(\eta_{1}\right)+(1-\chi) \varphi\left(\eta_{2}\right),
$$

holds for all $\eta_{1}, \eta_{2} \in I$ and $\chi \in[0,1]$.

The Hermite-Hadamard inequality states that if a mapping $\varphi: J \subset \mathbb{R} \rightarrow \mathbb{R}$ is convex on $J$ for $\eta_{1}, \eta_{2} \in J$ and $\eta_{2}>\eta_{1}$, then

$$
\varphi\left(\frac{\eta_{1}+\eta_{2}}{2}\right) \leq \frac{1}{\eta_{2}-\eta_{1}} \int_{\eta_{1}}^{\eta_{2}} \varphi(\chi) d \chi \leq \frac{\varphi\left(\eta_{1}\right)+\varphi\left(\eta_{2}\right)}{2} .
$$

Interested readers can refer to $[8,29]$.

Definition 2 ([30]). A function $\varphi:[0,+\infty) \rightarrow \mathbb{R}$ is said to be s-convex in the second sense for a real number $s \in(0,1]$ or $\varphi$ belongs to the class of $K_{s}^{2}$, if

$$
\varphi\left(\chi \eta_{1}+(1-\chi) \eta_{2}\right) \leq \chi^{s} \varphi\left(\eta_{1}\right)+(1-\chi)^{s} \varphi\left(\eta_{2}\right)
$$

holds for all $\eta_{1}, \eta_{2} \in[0,+\infty)$ and $\chi \in[0,1]$.

Breckner in his article [31] introduced s-convex functions. Hudzik presented several properties and connections with s-convexity in the first sense in [32]. Usually, when we put $s=1$ for $s$-convexity, it reduces to usual convexity. In [29], Dragomir et al. proved a generalized Hadamard's inequality, which holds for $s$-convex functions in the second sense.

Recently, many researchers have investigated the importance and development of the theory of exponentially convex functions. The fruitful importance of exponential-type convexity is that it can be used to manipulate for statistical learning, image processing, stochastic optimization and sequential prediction. In 2020, Kadakal and İşcan investigated a new class of exponential convexity, which is stated as follows: 
Definition 3 ([27]). A nonnegative real-valued function $\varphi: J \subset \mathbb{R} \rightarrow \mathbb{R}$ is known to be an exponential convex function if the following inequality holds:

$$
\varphi\left(\chi \eta_{1}+(1-\chi) \eta_{2}\right) \leq\left(e^{\chi}-1\right) \varphi\left(\eta_{1}\right)+\left(e^{(1-\chi)}-1\right) \varphi\left(\eta_{2}\right) .
$$

Definition 4 ([33]). A nonnegative real-valued function $\psi: I \rightarrow \mathbb{R}$ is called n-polynomial convex, if

$$
\psi\left(\chi \eta_{1}+(1-\chi) \eta_{2}\right) \leq \frac{1}{n} \sum_{i=1}^{n}\left[1-(1-\chi)^{i}\right] \psi\left(\eta_{1}\right)+\frac{1}{n} \sum_{i=1}^{n}\left[1-\chi^{i}\right] \psi\left(\eta_{2}\right),
$$

holds for every $\eta_{1}, \eta_{2} \in I, \chi \in[0,1], s \in[0,1]$ and $n \in \mathbb{N}$.

Definition 5 (see, for details, [6]). Let $\varphi \in \mathcal{L}\left[\eta_{1}, \eta_{2}\right]$. Then the fractional integrals $J_{\eta_{1}^{+}}^{\alpha}$ and $J_{\eta_{2}^{-}}^{\alpha}$ of order $\alpha>0$ are defined by

$$
J_{\eta_{1}^{+}}^{\alpha} \varphi(x):=\frac{1}{\Gamma(\alpha)} \int_{\eta_{1}}^{x}(x-\mathbf{z})^{\alpha-1} \varphi(\mathbf{z}) d \mathbf{z} \quad\left(0 \leqq \eta_{1}<x<\eta_{2}\right)
$$

and

$$
J_{\eta_{2}^{-}}^{\alpha} \varphi(x):=\frac{1}{\Gamma(\alpha)} \int_{x}^{\eta_{2}}(\mathbf{z}-x)^{\alpha-1} \varphi(\mathbf{z}) d \mathbf{z} \quad\left(0 \leqq \eta_{1}<x<\eta_{2}\right),
$$

respectively.

\section{Generalized Exponentially s-Convex Function}

Definition 6. Let $n \in \mathbb{N}$ and $s \in(0,1]$. Then the nonnegative real-valued function $\varphi: J \subset \mathbb{R} \rightarrow \mathbb{R}$ is known to be an n-polynomial exponentially s-convex function if the inequality holds:

$$
\varphi\left(\chi \eta_{1}+(1-\chi) \eta_{2}\right) \leq \frac{1}{n} \sum_{i=1}^{n}\left(e^{s \chi}-1\right)^{i} \varphi\left(\eta_{1}\right)+\frac{1}{n} \sum_{i=1}^{n}\left(e^{s(1-\chi)}-1\right)^{i} \varphi\left(\eta_{2}\right) .
$$

We represent the class of all n-polynomial exponentially type convex functions on the interval $J$ as POLEXPC $(J)$ for each $\eta_{1}, \eta_{2} \in J$ and $\chi \in[0,1]$.

Remark 1. In Definition 6, if $n=s=1$, then the 1-polynomial exponentially s-convex function reduces to the classical exponential-type convexity given by İscan in [27].

Remark 2. The range of the exponentially s-convex functions for some fixed $s \in[\ln 2.4,1]$ is $[0,+\infty)$.

Lemma 1. For all $\chi \in[0,1]$ and for some fixed $s \in[\ln 2.4,1]$ the following inequalities $\frac{1}{n} \sum_{i=1}^{n}\left(e^{s \chi}-1\right)^{i} \geq \chi^{s}$ and $\frac{1}{n} \sum_{i=1}^{n}\left(e^{s(1-\chi)}-1\right)^{i} \geq(1-\chi)^{s}$ hold.

Proof. Now, we will prove the first inequality i.e., $\frac{1}{n} \sum_{i=1}^{n}\left(e^{s \chi}-1\right)^{i} \geq \chi^{s}$ for all $\chi \in[0,1]$ and $s \in[\ln 2.4,1]$ and $n \in N$.

The following inequality is well-known as Bernoulli inequality in mathematical analysis

$$
\begin{aligned}
& \left(-1+e^{s \chi}\right)^{i} \geq 1+i e^{s \chi} \\
\Longrightarrow & -1+\left(-1+e^{s \chi}\right)^{i} \geq i e^{s \chi} \\
\Longrightarrow & \frac{-1+\left(-1+e^{s \chi}\right)^{i}}{i e^{s \chi}} \leq 1
\end{aligned}
$$


Thus, we have

$$
\begin{gathered}
\frac{1}{n} \sum_{i=1}^{n}\left(e^{s \chi}-1\right)^{i-1}=\frac{1-\left(-1+e^{s \chi}\right)^{n}}{n e^{s \chi}} \leq 1 \\
n\left(e^{s \chi}-1\right)\left[-1+\frac{1}{n} \sum_{i=1}^{n}\left(e^{s \chi}-1\right)^{i-1}\right]=-n\left(e^{s \chi}-1\right)+\frac{1}{n} \sum_{i=1}^{n}\left(e^{s \chi}-1\right)^{i} \leq 0
\end{gathered}
$$

Hence,

$$
\chi^{s} \leq \frac{1}{n} \sum_{i=1}^{n}\left(e^{s \chi}-1\right)^{i}
$$

Consequently, similar computation proves

$$
(1-\chi)^{s} \leq \frac{1}{n} \sum_{i=1}^{n}\left(e^{s(1-\chi)}-1\right)^{i}
$$

Proposition 1. Every nonnegative s-convex function is an n-polynomial exponentially s-convex function for $s \in[\ln 2.4,1]$.

Proof. Applying Lemma 1 and $s \in[\ln 2.4,1]$, we have

$$
\begin{aligned}
& \varphi\left(\chi \eta_{1}+(1-\chi) \eta_{2}\right) \leq \chi^{s} \varphi\left(\eta_{1}\right)+(1-\chi)^{s} \varphi\left(\eta_{2}\right) \\
\leq & \frac{1}{n} \sum_{i=1}^{n}\left(e^{s \chi}-1\right)^{i} \varphi\left(\eta_{1}\right)+\frac{1}{n} \sum_{i=1}^{n}\left(e^{(1-\chi) s}-1\right)^{i} \varphi\left(\eta_{2}\right) .
\end{aligned}
$$

Remark 3. If in the above proposition $s=1$, then every nonnegative convex function is an $n$-polynomial exponentially s-convex function for $s \in[\ln 2.4,1]$.

Now, we will make some examples in the support of the newly introduced function.

Example 1. Since, $\varphi(x)=e^{x}$ is a nonnegative convex function for all $x>0$. Using Remark 3 , it is also an n-polynomial exponentially s-convex function for $s \in[\ln 2.4,1]$.

Example 2. Since, $\varphi(x)=c$ is a nonnegative convex function on $R$ for any $c \geq 0$, using Remark 3 , it is also an n-polynomial exponentially s-convex function for $s \in[\ln 2.4,1]$.

Example 3. Since $\varphi(x)=\frac{1}{x}$ for all $x>0$, is a nonnegative convex function, using Remark 3 , it is also an n-polynomial exponentially s-convex function for $s \in[\ln 2.4,1]$.

Example 4. Since $\varphi(x)=\frac{q}{m+q} x^{\frac{m}{q}+1}$ for $m>1$ and $q \geq 1$, is a nonnegative convex function. Using Proposition 3, it is also an n-polynomial exponentially s-convex function for $s \in[\ln 2.4,1]$.

Example 5. Dragomir [29] clearly investigated and proved that the function $\varphi(x)=x^{l s}, x>0$ is an s-convex function, for the above-mentioned conditions $s \in(0,1)$ and $1 \leq l \leq \frac{1}{s}$. In addition, using Proposition 1, it is also an n-polynomial exponentially s-convex function for $s \in[\ln 2.4,1]$.

Remark 4. If we assign $n=2$ in Definition 7, we obtain the following definition for 2-polynomial exponentially s-convex function. 


$$
\varphi\left(\chi \eta_{1}+(1-\chi) \eta_{2}\right) \leq\left(\frac{e^{2 s \chi}-e^{s \chi}}{2}\right) \varphi\left(\eta_{1}\right)+\left(\frac{e^{2 s(1-\chi)-e^{s(1-\chi)}}}{2}\right) \varphi\left(\eta_{2}\right) .
$$

\section{Hermite-Hadamard Type Inequality via Fractional Operator}

In this Section, we present one Hermite-Hadamard-type inequality for the $n$-polynomial exponentially $s$-convex function.

Theorem 1. Let $\varphi: \mathbb{A}=\left[\eta_{1}, \eta_{2}\right] \rightarrow \mathbb{R}$ be a positive function with $0 \leq \eta_{1} \leq \eta_{2}$ and $\varphi$ be an integrable function on the closed interval sets $\eta_{1}$ and $\eta_{2}$. If $\varphi$ is an n-polynomial exponentially s-convex function, then the following inequality for fractional integral with $\alpha>0$ and $s \in[\ln 2.4,1]$ holds:

$$
\begin{aligned}
& \frac{1}{\frac{1}{n} \sum_{i=1}^{n}\left(e^{\frac{s}{2}}-1\right)^{i}} \leq \frac{\Gamma(\alpha+1)}{\left(\eta_{2}-\eta_{1}\right)^{\alpha}}\left[J_{\eta_{1}^{+}}^{\alpha} \varphi\left(\eta_{2}\right)+J_{\eta_{2}^{-}}^{\alpha} \varphi\left(\eta_{1}\right)\right] \\
& \leq \alpha\left[\varphi\left(\eta_{1}\right)+\varphi\left(\eta_{2}\right)\right] \int_{0}^{1} \chi^{\alpha-1}\left\{\frac{1}{n} \sum_{i=1}^{n}\left(e^{s \chi}-1\right)^{i}+\frac{1}{n} \sum_{i=1}^{n}\left(e^{s(1-\chi)}-1\right)^{i}\right\} d \chi .
\end{aligned}
$$

Proof. Let $z_{1}, z_{2} \in \mathbb{A}$. Then, using the definition of $n$-polynomial exponentially $s$-convex function $\varphi$ on $\mathbb{A}$, we have

$$
\varphi\left(\frac{z_{1}+z_{2}}{2}\right) \leq \frac{1}{n} \sum_{i=1}^{n}\left(e^{\frac{s}{2}}-1\right)^{i}\left[\varphi\left(z_{1}\right)+\varphi\left(z_{2}\right)\right]
$$

$$
\text { Suppose } z_{1}=\chi \eta_{2}+(1-\chi) \eta_{1} \text { and } z_{2}=\chi \eta_{1}+(1-\chi) \eta_{2} \text {. }
$$

Then (10) leads to

$$
\varphi\left(\frac{\eta_{1}+\eta_{2}}{2}\right) \leq \frac{1}{n} \sum_{i=1}^{n}\left(e^{\frac{s}{2}}-1\right)^{i}\left[\varphi\left(\chi \eta_{2}+(1-\chi) \eta_{1}\right)+\varphi\left(\chi \eta_{1}+(1-\chi) \eta_{2}\right)\right] .
$$

Now, multiplying both sides of (11) by $\chi^{\alpha-1}$ and then, integrating the resultant inequality with respect to $\chi$ over $[0,1]$ and, we obtain

$$
\frac{1}{\alpha} \varphi\left(\frac{\eta_{1}+\eta_{2}}{2}\right) \leq \frac{1}{n} \sum_{i=1}^{n}\left(e^{\frac{s}{2}}-1\right)^{i}\left[\int_{0}^{1} \chi^{\alpha-1} \varphi\left(\chi \eta_{2}+(1-\chi) \eta_{1}\right) d \chi+\int_{0}^{1} \chi^{\alpha-1} \varphi\left(\chi \eta_{1}+(1-\chi) \eta_{2}\right) d \chi\right]
$$

Hence, we obtain

$$
\frac{1}{\frac{1}{n} \sum_{i=1}^{n}\left(e^{\frac{s}{2}}-1\right)^{i}} \varphi\left(\frac{\eta_{1}+\eta_{2}}{2}\right) \leq \frac{\Gamma(\alpha+1)}{\left(\eta_{2}-\eta_{1}\right)^{\alpha}}\left[J_{\eta_{1}^{+}}^{\alpha} \varphi\left(\eta_{2}\right)+J_{\eta_{2}^{-}}^{\alpha} \varphi\left(\eta_{1}\right)\right] .
$$

The proof of the first part of the inequality (9) is complete.

Next, we prove the second part of the inequality (9) using the fact that $\varphi$ is an exponentially s-convex function, we obtain

$$
\varphi\left(\chi \eta_{2}+(1-\chi) \eta_{1}\right) \leq \frac{1}{n} \sum_{i=1}^{n}\left(e^{s \chi}-1\right)^{i} \varphi\left(\eta_{2}\right)+\frac{1}{n} \sum_{i=1}^{n}\left(e^{s(1-\chi)}-1\right)^{i} \varphi\left(\eta_{1}\right)
$$

and

$$
\varphi\left(\chi \eta_{1}+(1-\chi) \eta_{2}\right) \leq \frac{1}{n} \sum_{i=1}^{n}\left(e^{s \chi}-1\right)^{i} \varphi\left(\eta_{1}\right)+\frac{1}{n} \sum_{i=1}^{n}\left(e^{s(1-\chi)}-1\right)^{i} \varphi\left(\eta_{2}\right) .
$$


Upon adding the above inequalities, we obtain

$$
\begin{aligned}
& \varphi\left(\chi \eta_{2}+(1-\chi) \eta_{1}\right)+\varphi\left(\chi \eta_{1}+(1-\chi) \eta_{2}\right) \\
& \leq\left[\varphi\left(\eta_{1}\right)+\varphi\left(\eta_{2}\right)\right]\left\{\frac{1}{n} \sum_{i=1}^{n}\left(e^{s \chi}-1\right)^{i}+\frac{1}{n} \sum_{i=1}^{n}\left(e^{s(1-\chi)}-1\right)^{i}\right\} .
\end{aligned}
$$

Now, multiplying both sides of (14) by $\chi^{\alpha-1}$, integrating the resultant inequality with respect to $\chi$ over $[0,1]$ and then using the change of variable technique, we obtain

$$
\begin{aligned}
& \frac{\Gamma(\alpha)}{\left(\eta_{2}-\eta_{1}\right)^{\alpha}}\left[J_{\eta_{1}^{+}}^{\alpha} \varphi\left(\eta_{2}\right)+J_{\eta_{2}^{-}}^{\alpha} \varphi\left(\eta_{1}\right)\right] \\
& \leq\left[\varphi\left(\eta_{1}\right)+\varphi\left(\eta_{2}\right)\right] \int_{0}^{1} \chi^{\alpha-1}\left\{\frac{1}{n} \sum_{i=1}^{n}\left(e^{s \chi}-1\right)^{i}+\frac{1}{n} \sum_{i=1}^{n}\left(e^{s(1-\chi)}-1\right)^{i}\right\} d \chi,
\end{aligned}
$$

Consequently,

$$
\begin{aligned}
& \frac{\Gamma(\alpha+1)}{\left(\eta_{2}-\eta_{1}\right)^{\alpha}}\left[J_{\eta_{1}^{+}}^{\alpha} \varphi\left(\eta_{2}\right)+J_{\eta_{2}^{-}}^{\alpha} \varphi\left(\eta_{1}\right)\right] \\
& \leq \alpha\left[\varphi\left(\eta_{1}\right)+\varphi\left(\eta_{2}\right)\right] \int_{0}^{1} \chi^{\alpha-1}\left\{\frac{1}{n} \sum_{i=1}^{n}\left(e^{s \chi}-1\right)^{i}+\frac{1}{n} \sum_{i=1}^{n}\left(e^{s(1-\chi)}-1\right)^{i}\right\} d \chi .
\end{aligned}
$$

This completes rest of the proof.

Remark 5. Exclusively, in Theorem 1, If we assign $\alpha=1$, then we attain

$$
\begin{aligned}
& \frac{1}{2 \frac{1}{n} \sum_{i=1}^{n}\left(e^{\frac{s}{2}}-1\right)^{i}} \varphi\left(\frac{\eta_{1}+\eta_{2}}{2}\right) \leq \frac{1}{\eta_{2}-\eta_{1}} \int_{\eta_{1}}^{\eta_{2}} \varphi(\chi) d \chi \\
& \leq \frac{1}{n} \sum_{i=1}^{n}\left(\frac{e^{s}-s-1}{s}\right)^{i}\left[\varphi\left(\eta_{1}\right)+\varphi\left(\eta_{2}\right)\right] .
\end{aligned}
$$

Remark 6. Exclusively, in Theorem 1, If we assign $n=s=\alpha=1$, then it reduces to [Theorem 3.1, [27]].

In the next section, we establish new Ostrowski-type inequalities for $n$-polynomial exponentially $s-$ convexity via Riemann-Liouville fractional integral. A useful and interesting feature of our results is that they provide new estimates on these type of inequalities for fractional integrals.

\section{Ostrowski-Type Inequalities for $n$-Polynomial Exponentially $s-$ Convexity via Fractional Integral}

To prove our results, we need the following identity (see $[34,35])$.

Lemma 2. Suppose a mapping $\varphi: J \subseteq R \rightarrow R$ is differentiable on $J^{0}$, where $\eta_{1}, \eta_{2} \in J$ with $\eta_{1}<\eta_{2}$. If $\varphi^{\prime} \in \mathcal{L}\left[\eta_{1}, \eta_{2}\right]$, for all $z \in\left[\eta_{1}, \eta_{2}\right]$ and $\alpha>0$, then the following equality holds:

$$
\begin{aligned}
& \left(\frac{\left(z-\eta_{1}\right)^{\alpha}+\left(\eta_{2}-z\right)^{\alpha}}{\eta_{2}-\eta_{1}}\right) \varphi(z)-\frac{\Gamma(\alpha+1)}{\eta_{2}-\eta_{1}}\left\{J_{z^{-}}^{\alpha} \varphi\left(\eta_{1}\right)+J_{z^{+}}^{\alpha} \varphi\left(\eta_{2}\right)\right\} \\
& =\frac{\left(z-\eta_{1}\right)^{\alpha+1}}{\eta_{2}-\eta_{1}} \int_{0}^{1} \chi^{\alpha} \varphi^{\prime}\left(\chi z+(1-\chi) \eta_{1}\right) d \chi-\frac{\left(\eta_{2}-z\right)^{\alpha+1}}{\eta_{2}-\eta_{1}} \int_{0}^{1} \chi^{\alpha} \varphi^{\prime}\left(\chi z+(1-\chi) \eta_{2}\right) d \chi,
\end{aligned}
$$

where $\Gamma$ is the Euler gamma function. 
Theorem 2. Suppose a mapping $\varphi: J \subseteq R \rightarrow R$ is differentiable on $J^{o}$, where $\eta_{1}, \eta_{2} \in J$ with $\eta_{1}<\eta_{2}$. If $\left|\varphi^{\prime}\right|$ is n-polynomial exponentially $s$-convex on $\left[\eta_{1}, \eta_{2}\right]$ for some $s \in(0,1]$, $\varphi^{\prime} \in \mathcal{L}\left[\eta_{1}, \eta_{2}\right]$ and $\left|\varphi^{\prime}(z)\right| \leq K$, for all $z \in\left[\eta_{1}, \eta_{2}\right], \alpha>0$, then the following inequality holds:

$$
\begin{aligned}
& \left|\left(\frac{\left(z-\eta_{1}\right)^{\alpha}+\left(\eta_{2}-z\right)^{\alpha}}{\eta_{2}-\eta_{1}}\right) \varphi(z)-\frac{\Gamma(\alpha+1)}{\eta_{2}-\eta_{1}}\left\{J_{z^{-}}^{\alpha} \varphi\left(\eta_{1}\right)+J_{z^{+}}^{\alpha} \varphi\left(\eta_{2}\right)\right\}\right| \\
& \leq \frac{K}{n\left(\eta_{2}-\eta_{1}\right)} \\
& \times\left[( z - \eta _ { 1 } ) ^ { \alpha + 1 } \left\{\sum_{i=1}^{n}\left(\frac{\gamma(\alpha+1,-s)-\Gamma(\alpha+1)}{(-s)^{\alpha} s}-\frac{1}{\alpha+1}\right)^{i}\right.\right. \\
& \left.-\sum_{i=1}^{n}\left(\frac{(\gamma(\alpha+1, s)-\Gamma(\alpha+1)) e^{s}}{s^{\alpha+1}}+\frac{1}{\alpha+1}\right)^{i}\right\} \\
& +\left(\eta_{2}-z\right)^{\alpha+1}\left\{\sum_{i=1}^{n}\left(\frac{\gamma(\alpha+1,-s)-\Gamma(\alpha+1)}{(-s)^{\alpha} s}-\frac{1}{\alpha+1}\right)^{i}\right. \\
& \left.\left.-\sum_{i=1}^{n}\left(\frac{(\gamma(\alpha+1, s)-\Gamma(\alpha+1)) e^{s}}{s^{\alpha+1}}+\frac{1}{\alpha+1}\right)^{i}\right\}\right] .
\end{aligned}
$$

Proof. From Lemma 2, n-polynomial exponentially $s$-convexity of $\left|\varphi^{\prime}\right|$ and $\left|\varphi^{\prime}(z)\right| \leq K$, we have

$$
\begin{aligned}
& \left|\left(\frac{\left(z-\eta_{1}\right)^{\alpha}+\left(\eta_{2}-z\right)^{\alpha}}{\eta_{2}-\eta_{1}}\right) \varphi(z)-\frac{\Gamma(\alpha+1)}{\eta_{2}-\eta_{1}}\left\{J_{z^{-}}^{\alpha} \varphi\left(\eta_{1}\right)+J_{z^{+}}^{\alpha} \varphi\left(\eta_{2}\right)\right\}\right| \\
& \leq \frac{\left(z-\eta_{1}\right)^{\alpha+1}}{\eta_{2}-\eta_{1}} \int_{0}^{1} \chi^{\alpha}\left|\varphi^{\prime}\left(\chi z+(1-\chi) \eta_{1}\right)\right| d \chi+\frac{\left(\eta_{2}-z\right)^{\alpha+1}}{\eta_{2}-\eta_{1}} \int_{0}^{1} \chi^{\alpha}\left|\varphi^{\prime}\left(\chi z+(1-\chi) \eta_{2}\right)\right| d \chi \\
& \leq \frac{\left(z-\eta_{1}\right)^{\alpha+1}}{\eta_{2}-\eta_{1}} \int_{0}^{1} \chi^{\alpha}\left\{\frac{1}{n} \sum_{i=1}^{n}\left(e^{s \chi}-1\right)^{i}\left|\varphi^{\prime}(z)\right|+\frac{1}{n} \sum_{i=1}^{n}\left(e^{s(1-\chi)}-1\right)^{i}\left|\varphi^{\prime}\left(\eta_{1}\right)\right|\right\} d \chi \\
& +\frac{\left(\eta_{2}-z\right)^{\alpha+1}}{\eta_{2}-\eta_{1}} \int_{0}^{1} \chi^{\alpha}\left\{\frac{1}{n} \sum_{i=1}^{n}\left(e^{s \chi}-1\right)^{i}\left|\varphi^{\prime}(z)\right|+\frac{1}{n} \sum_{i=1}^{n}\left(e^{s(1-\chi)}-1\right)^{i}\left|\varphi^{\prime}\left(\eta_{1}\right)\right|\right\} d \chi \\
& \leq \frac{\left(z-\eta_{1}\right)^{\alpha+1}}{\eta_{2}-\eta_{1}}\left\{\left|\varphi^{\prime}(z)\right| \int_{0}^{1} \chi^{\alpha} \frac{1}{n} \sum_{i=1}^{n}\left(e^{s \chi}-1\right)^{i} d \chi+\left|\varphi^{\prime}\left(\eta_{1}\right)\right| \int_{0}^{1} \chi^{\alpha} \frac{1}{n} \sum_{i=1}^{n}\left(e^{s(1-\chi)}-1\right)^{i} d \chi\right\} \\
& +\frac{\left(\eta_{2}-z\right)^{\alpha+1}}{\eta_{2}-\eta_{1}}\left\{\left|\varphi^{\prime}(z)\right| \int_{0}^{1} \chi^{\alpha} \frac{1}{n} \sum_{i=1}^{n}\left(e^{s \chi}-1\right)^{i} d \chi+\left|\varphi^{\prime}\left(\eta_{2}\right)\right| \int_{0}^{1} \chi^{\alpha} \frac{1}{n} \sum_{i=1}^{n}\left(e^{s(1-\chi)}-1\right)^{i} d \chi\right\} \\
& \leq \frac{K}{n\left(\eta_{2}-\eta_{1}\right)} \\
& \times\left(z-\eta_{1}\right)^{\alpha+1}\left\{\sum_{i=1}^{n}\left(\frac{\gamma(\alpha+1,-s)-\Gamma(\alpha+1)}{(-s)^{\alpha} s}-\frac{1}{\alpha+1}\right)^{i}\right. \\
& \left.-\sum_{i=1}^{n}\left(\frac{(\gamma(\alpha+1, s)-\Gamma(\alpha+1)) e^{s}}{s^{\alpha+1}}+\frac{1}{\alpha+1}\right)^{i}\right\} . \\
& \left.+\sum_{i=1}^{n}\left(\frac{(\gamma(\alpha+1, s)-\Gamma(\alpha+1)) e^{s}}{s^{\alpha+1}}+\frac{1}{\alpha+1}\right)^{i}\right\} \\
& +\frac{K}{n\left(\eta_{2}-\eta_{1}\right)} \\
& \times\left(\eta_{2}-z\right)^{\alpha+1}\left\{\sum_{i=1}^{n}\left(\frac{\gamma(\alpha+1,-s)-\Gamma(\alpha+1)}{\alpha+1}\right)^{i}\right. \\
& +\frac{1}{\alpha} s
\end{aligned}
$$

After further simplifications, proof of Theorem 17 will be completed. 
Corollary 1. If we assign $n=1$ in Theorem 2, then

$$
\begin{aligned}
& \left|\left(\frac{\left(z-\eta_{1}\right)^{\alpha}+\left(\eta_{2}-z\right)^{\alpha}}{\eta_{2}-\eta_{1}}\right) \varphi(z)-\frac{\Gamma(\alpha+1)}{\eta_{2}-\eta_{1}}\left\{J_{z^{-}}^{\alpha} \varphi\left(\eta_{1}\right)+J_{z^{+}}^{\alpha} \varphi\left(\eta_{2}\right)\right\}\right| \\
& \leq \frac{K}{\left(\eta_{2}-\eta_{1}\right)} \\
& \times\left[( z - \eta _ { 1 } ) ^ { \alpha + 1 } \left\{\left(\frac{\gamma(\alpha+1,-s)-\Gamma(\alpha+1)}{(-s)^{\alpha} s}-\frac{1}{\alpha+1}\right)\right.\right. \\
& -\left(\frac{(\gamma(\alpha+1, s)-\Gamma(\alpha+1)) e^{s}}{\left.\left.s^{\alpha+1}+\frac{1}{\alpha+1}\right)\right\}}\right. \\
& +\left(\eta_{2}-z\right)^{\alpha+1}\left\{\left(\frac{\gamma(\alpha+1,-s)-\Gamma(\alpha+1)}{(-s)^{\alpha} s}-\frac{1}{\alpha+1}\right)\right. \\
& \left.\left.-\left(\frac{(\gamma(\alpha+1, s)-\Gamma(\alpha+1)) e^{s}}{s^{\alpha+1}}+\frac{1}{\alpha+1}\right)\right\}\right] .
\end{aligned}
$$

Corollary 2. If we assign $s=1$ in Theorem 2, then

$$
\begin{aligned}
& \left|\left(\frac{\left(z-\eta_{1}\right)^{\alpha}+\left(\eta_{2}-z\right)^{\alpha}}{\eta_{2}-\eta_{1}}\right) \varphi(z)-\frac{\Gamma(\alpha+1)}{\eta_{2}-\eta_{1}}\left\{J_{z^{-}}^{\alpha} \varphi\left(\eta_{1}\right)+J_{z^{+}}^{\alpha} \varphi\left(\eta_{2}\right)\right\}\right| \\
& \leq \frac{K}{n\left(\eta_{2}-\eta_{1}\right)} \\
& \times\left[( z - \eta _ { 1 } ) ^ { \alpha + 1 } \left\{\sum_{i=1}^{n}\left(\frac{\gamma(\alpha+1,-1)-\Gamma(\alpha+1)}{(-1)^{\alpha}}-\frac{1}{\alpha+1}\right)^{i}\right.\right. \\
& \left.-\sum_{i=1}^{n}\left((\gamma(\alpha+1,1)-\Gamma(\alpha+1)) e+\frac{1}{\alpha+1}\right)^{i}\right\} \\
& +\left(\eta_{2}-z\right)^{\alpha+1}\left\{\sum_{i=1}^{n}\left(\frac{\gamma(\alpha+1,-1)-\Gamma(\alpha+1)}{(-1)^{\alpha}}-\frac{1}{\alpha+1}\right)^{i}\right. \\
& \left.\left.-\sum_{i=1}^{n}\left((\gamma(\alpha+1,1)-\Gamma(\alpha+1)) e+\frac{1}{\alpha+1}\right)^{i}\right\}\right] .
\end{aligned}
$$

Corollary 3. If we assign $\alpha=1$ in Theorem 2, then

$$
\begin{aligned}
& \left|\varphi(z)-\frac{1}{\eta_{2}-\eta_{1}} \int_{\eta_{1}}^{\eta_{2}} \varphi(\chi) d \chi\right| \\
& \leq \frac{K}{\left(\eta_{2}-\eta_{1}\right) n}\left[\left(z-\eta_{1}\right)^{2}\left\{\sum_{i=1}^{n}\left(\frac{2+2(s-1) e^{s}-s^{2}}{2 s^{2}}\right)^{i}+\sum_{i=1}^{n}\left(\frac{2 e^{s}-s^{2}-2 s-2}{2 s^{2}}\right)^{i}\right\}\right. \\
& \left.+\left(\eta_{2}-z\right)^{2}\left\{\sum_{i=1}^{n}\left(\frac{2+2(s-1) e^{s}-s^{2}}{2 s^{2}}\right)^{i}+\sum_{i=1}^{n}\left(\frac{2 e^{s}-s^{2}-2 s-2}{2 s^{2}}\right)^{i}\right\}\right] .
\end{aligned}
$$

Corollary 4. If we assign $\alpha=1$ and $z=\eta_{1}$ in Theorem 2, then

$$
\begin{aligned}
& \left|\varphi\left(\eta_{1}\right)-\frac{1}{\eta_{2}-\eta_{1}} \int_{\eta_{1}}^{\eta_{2}} \varphi(\chi) d \chi\right| \\
& \leq \frac{K}{\left(\eta_{2}-\eta_{1}\right) n}\left[\left(\eta_{2}-\eta_{1}\right)^{2}\left\{\sum_{i=1}^{n}\left(\frac{2+2(s-1) e^{s}-s^{2}}{2 s^{2}}\right)^{i}+\sum_{i=1}^{n}\left(\frac{2 e^{s}-s^{2}-2 s-2}{2 s^{2}}\right)^{i}\right\}\right] .
\end{aligned}
$$


Corollary 5. If we assign $\alpha=1$ and $z=\eta_{2}$ in Theorem 2, then

$$
\begin{aligned}
& \left|\varphi\left(\eta_{2}\right)-\frac{1}{\eta_{2}-\eta_{1}} \int_{\eta_{1}}^{\eta_{2}} \varphi(\chi) d \chi\right| \\
& \leq \frac{K}{\left(\eta_{2}-\eta_{1}\right) n}\left[\left(\eta_{2}-\eta_{1}\right)^{2}\left\{\sum_{i=1}^{n}\left(\frac{2+2(s-1) e^{s}-s^{2}}{2 s^{2}}\right)^{i}+\sum_{i=1}^{n}\left(\frac{2 e^{s}-s^{2}-2 s-2}{2 s^{2}}\right)^{i}\right\}\right] .
\end{aligned}
$$

Theorem 3. Suppose a mapping $\varphi: J \subseteq R \rightarrow R$ is differentiable on $J^{o}$, where $\eta_{1}, \eta_{2} \in J$ with $\eta_{1}<\eta_{2}$. If $\left|\varphi^{\prime}\right|^{q}$ is n-polynomial exponentially $s$-convex on $\left[\eta_{1}, \eta_{2}\right]$ for some $s \in(0,1], q>1$, $q^{-1}=1-p^{-1}, \varphi^{\prime} \in \mathcal{L}\left[\eta_{1}, \eta_{2}\right]$ and $\left|\varphi^{\prime}(z)\right| \leq K$, for all $z \in\left[\eta_{1}, \eta_{2}\right]$, with $\alpha>0$, then the following inequality holds:

$$
\begin{aligned}
& \left|\left(\frac{\left(z-\eta_{1}\right)^{\alpha}+\left(\eta_{2}-z\right)^{\alpha}}{\eta_{2}-\eta_{1}}\right) \varphi(z)-\frac{\Gamma(\alpha+1)}{\eta_{2}-\eta_{1}}\left\{J_{z^{-}}^{\alpha} \varphi\left(\eta_{1}\right)+J_{z^{+}}^{\alpha} \varphi\left(\eta_{2}\right)\right\}\right| \\
& \leq \frac{2^{\frac{1}{q}} K}{\sqrt[q]{n}\left(\eta_{2}-\eta_{1}\right)}\left(\frac{1}{\alpha p+1}\right)^{\frac{1}{p}} \\
& \times\left[\left(z-\eta_{1}\right)^{\alpha+1}\left\{\sum_{i=1}^{n}\left(\frac{e^{s}-s-1}{s}\right)^{i}\right\}^{\frac{1}{q}}+\left(\eta_{2}-z\right)^{\alpha+1}\left\{\sum_{i=1}^{n}\left(\frac{e^{s}-s-1}{s}\right)^{i}\right\}^{\frac{1}{q}}\right] .
\end{aligned}
$$

Proof. Applying Lemma 2 and the well-known Hölder's inequality, we have

$$
\begin{aligned}
& \left|\left(\frac{\left(z-\eta_{1}\right)^{\alpha}+\left(\eta_{2}-z\right)^{\alpha}}{\eta_{2}-\eta_{1}}\right) \varphi(z)-\frac{\Gamma(\alpha+1)}{\eta_{2}-\eta_{1}}\left\{J_{z^{-}}^{\alpha} \varphi\left(\eta_{1}\right)+J_{z^{+}}^{\alpha} \varphi\left(\eta_{2}\right)\right\}\right| \\
& \leq \frac{\left(z-\eta_{1}\right)^{\alpha+1}}{\eta_{2}-\eta_{1}} \int_{0}^{1} \chi^{\alpha}\left|\varphi^{\prime}\left(\chi z+(1-\chi) \eta_{1}\right)\right| d \chi+\frac{\left(\eta_{2}-z\right)^{\alpha+1}}{\eta_{2}-\eta_{1}} \int_{0}^{1} \chi^{\alpha}\left|\varphi^{\prime}\left(\chi z+(1-\chi) \eta_{2}\right)\right| d \chi \\
& \leq \frac{\left(z-\eta_{1}\right)^{\alpha+1}}{\eta_{2}-\eta_{1}}\left(\int_{0}^{1} \chi^{\alpha p} d \chi\right)^{\frac{1}{p}}\left(\int_{0}^{1}\left|\varphi^{\prime}\left(\chi z+(1-\chi) \eta_{1}\right)\right|^{q} d \chi\right)^{\frac{1}{q}} \\
& +\frac{\left(\eta_{2}-z\right)^{\alpha+1}}{\eta_{2}-\eta_{1}}\left(\int_{0}^{1} \chi^{\alpha p} d \chi\right)^{\frac{1}{p}}\left(\int_{0}^{1}\left|\varphi^{\prime}\left(\chi z+(1-\chi) \eta_{2}\right)\right|^{q} d \chi\right)^{\frac{1}{q}} .
\end{aligned}
$$

Since $\left|\varphi^{\prime}\right|^{q}$ is $n$-polynomial exponentially $s$-convex and $\left|\varphi^{\prime}(z)\right| \leq K$, we obtain

$$
\begin{aligned}
& \int_{0}^{1}\left|\varphi^{\prime}\left(\chi z+(1-\chi) \eta_{1}\right)\right|^{q} d \chi=\int_{0}^{1}\left\{\frac{1}{n} \sum_{i=1}^{n}\left(e^{s \chi}-1\right)^{i}\left|\varphi^{\prime}(z)\right|^{q}+\frac{1}{n} \sum_{i=1}^{n}\left(e^{s(1-\chi)}-1\right)^{i}\left|\varphi^{\prime}\left(\eta_{1}\right)\right|^{q}\right\} d \chi \\
& \leq K^{q} \frac{1}{n} \sum_{i=1}^{n}\left(\frac{e^{s}-s-1}{s}\right)^{i}+K^{q} \frac{1}{n} \sum_{i=1}^{n}\left(\frac{e^{s}-s-1}{s}\right)^{i} \\
& \leq 2 K^{q} \frac{1}{n} \sum_{i=1}^{n}\left(\frac{e^{s}-s-1}{s}\right)^{i}
\end{aligned}
$$

and

$$
\begin{aligned}
& \int_{0}^{1}\left|\varphi^{\prime}\left(\chi z+(1-\chi) \eta_{2}\right)\right|^{q} d \chi=\int_{0}^{1}\left\{\frac{1}{n} \sum_{i=1}^{n}\left(e^{s \chi}-1\right)^{i}\left|\varphi^{\prime}(z)\right|^{q}+\frac{1}{n} \sum_{i=1}^{n}\left(e^{s(1-\chi)}-1\right)^{i}\left|\varphi^{\prime}\left(\eta_{2}\right)\right|^{q}\right\} d \chi \\
& \leq K^{q} \frac{1}{n} \sum_{i=1}^{n}\left(\frac{e^{s}-s-1}{s}\right)^{i}+K^{q} \frac{1}{n} \sum_{i=1}^{n}\left(\frac{e^{s}-s-1}{s}\right)^{i} \\
& \leq 2 K^{q} \frac{1}{n} \sum_{i=1}^{n}\left(\frac{e^{s}-s-1}{s}\right)^{i} .
\end{aligned}
$$

By connecting (20) and (21) with (19), we have the desired inequality (18). 
Corollary 6. If we assign $n=1$ in Theorem 3 , then

$$
\begin{aligned}
& \left|\left(\frac{\left(z-\eta_{1}\right)^{\alpha}+\left(\eta_{2}-z\right)^{\alpha}}{\eta_{2}-\eta_{1}}\right) \varphi(z)-\frac{\Gamma(\alpha+1)}{\eta_{2}-\eta_{1}}\left\{J_{z^{-}}^{\alpha} \varphi\left(\eta_{1}\right)+J_{z^{+}}^{\alpha} \varphi\left(\eta_{2}\right)\right\}\right| \\
& \leq \frac{2^{\frac{1}{q}} K}{\left(\eta_{2}-\eta_{1}\right)}\left(\frac{1}{\alpha p+1}\right)^{\frac{1}{p}} \\
& \times\left[\left(z-\eta_{1}\right)^{\alpha+1}\left(\frac{e^{s}-s-1}{s}\right)^{\frac{1}{q}}+\left(\eta_{2}-z\right)^{\alpha+1}\left(\frac{e^{s}-s-1}{s}\right)^{\frac{1}{q}}\right] .
\end{aligned}
$$

Corollary 7. If we assign $s=1$, in Theorem 3 , then

$$
\begin{aligned}
& \left|\left(\frac{\left(z-\eta_{1}\right)^{\alpha}+\left(\eta_{2}-z\right)^{\alpha}}{\eta_{2}-\eta_{1}}\right) \varphi(z)-\frac{\Gamma(\alpha+1)}{\eta_{2}-\eta_{1}}\left\{J_{z^{-}}^{\alpha} \varphi\left(\eta_{1}\right)+J_{z^{+}}^{\alpha} \varphi\left(\eta_{2}\right)\right\}\right| \\
& \leq \frac{2^{\frac{1}{q}} K}{\sqrt[q]{n}\left(\eta_{2}-\eta_{1}\right)}\left(\frac{1}{\alpha p+1}\right)^{\frac{1}{p}}\left[\left(z-\eta_{1}\right)^{\alpha+1}\left\{\sum_{i=1}^{n}(e-2)^{i}\right\}^{\frac{1}{\bar{q}}}+\left(\eta_{2}-z\right)^{\alpha+1}\left\{\sum_{i=1}^{n}(e-2)^{i}\right\}^{\frac{1}{q}}\right] .
\end{aligned}
$$

Corollary 8. If we assign $\alpha=1$, in Theorem 3, then

$$
\begin{aligned}
& \left|\varphi(z)-\frac{1}{\eta_{2}-\eta_{1}} \int_{\eta_{1}}^{\eta_{2}} \varphi(\chi) d \chi\right| \\
& \leq \frac{2^{\frac{1}{q}} K}{\left(\eta_{2}-\eta_{1}\right) \sqrt[q]{n}}\left(\frac{1}{p+1}\right)^{\frac{1}{p}}\left[\left(z-\eta_{1}\right)^{2}\left\{\sum_{i=1}^{n}\left(\frac{e^{s}-s-1}{s}\right)^{i}\right\}^{\frac{1}{q}}+\left(\eta_{2}-z\right)^{2}\left\{\sum_{i=1}^{n}\left(\frac{e^{s}-s-1}{s}\right)^{i}\right\}^{\frac{1}{q}}\right] .
\end{aligned}
$$

Corollary 9. If we assign $\alpha=1$ and $z=\eta_{1}$ in Theorem 3, then

$$
\begin{aligned}
& \left|\varphi\left(\eta_{1}\right)-\frac{1}{\eta_{2}-\eta_{1}} \int_{\eta_{1}}^{\eta_{2}} \varphi(\chi) d \chi\right| \\
& \leq \frac{2^{\frac{1}{q}} K}{\left(\eta_{2}-\eta_{1}\right) \sqrt[q]{n}}\left(\frac{1}{p+1}\right)^{\frac{1}{p}}\left[\left(\eta_{2}-\eta_{1}\right)^{2}\left\{\sum_{i=1}^{n}\left(\frac{e^{s}-s-1}{s}\right)^{i}\right\}^{\frac{1}{q}}\right] .
\end{aligned}
$$

Corollary 10. If we assign $\alpha=1$ and $z=\eta_{2}$ in Theorem 3 , then

$$
\begin{aligned}
& \left|\varphi\left(\eta_{2}\right)-\frac{1}{\eta_{2}-\eta_{1}} \int_{\eta_{1}}^{\eta_{2}} \varphi(\chi) d \chi\right| \\
& \leq \frac{2^{\frac{1}{q}} K}{\left(\eta_{2}-\eta_{1}\right) \sqrt[q]{n}}\left(\frac{1}{p+1}\right)^{\frac{1}{p}}\left[\left(\eta_{2}-\eta_{1}\right)^{2}\left\{\sum_{i=1}^{n}\left(\frac{e^{s}-s-1}{s}\right)^{i}\right\}^{\frac{1}{q}}\right] .
\end{aligned}
$$

Theorem 4. Suppose a mapping $\varphi: J \subseteq R \rightarrow R$ is differentiable on $J^{o}$, where $\eta_{1}, \eta_{2} \in J$ with $\eta_{1}<\eta_{2}$. Let $q \geq 1$ and $q^{-1}=1-p^{-1}$. If $\left|\varphi^{\prime}\right|^{q}$ is $n$-polynomial exponentially $s-$ convex on 
$\left[\eta_{1}, \eta_{2}\right]$ for some $s \in(0,1], \varphi^{\prime} \in \mathcal{L}\left[\eta_{1}, \eta_{2}\right]$ and $\left|\varphi^{\prime}(z)\right| \leq K$, for all $z \in\left[\eta_{1}, \eta_{2}\right]$, with $\alpha>0$, then the following inequality holds:

$$
\begin{aligned}
& \left|\left(\frac{\left(z-\eta_{1}\right)^{\alpha}+\left(\eta_{2}-z\right)^{\alpha}}{\eta_{2}-\eta_{1}}\right) \varphi(z)-\frac{\Gamma(\alpha+1)}{\eta_{2}-\eta_{1}}\left\{J_{z^{-}}^{\alpha} \varphi\left(\eta_{1}\right)+J_{z^{+}}^{\alpha} \varphi\left(\eta_{2}\right)\right\}\right| \\
& \leq \frac{K}{\sqrt[q]{n}\left(\eta_{2}-\eta_{1}\right)}\left(\frac{1}{\alpha+1}\right)^{1-\frac{1}{q}} \\
& \times\left[( z - \eta _ { 1 } ) ^ { \alpha + 1 } \left\{\sum_{i=1}^{n}\left(\frac{\gamma(\alpha+1,-s)-\Gamma(\alpha+1)}{(-s)^{\alpha} s}-\frac{1}{\alpha+1}\right)^{i}\right.\right. \\
& \left.-\sum_{i=1}^{n}\left((\gamma(\alpha+1, s)-\Gamma(\alpha+1)) s^{-\alpha-1} e^{s}-\frac{1}{\alpha+1}\right)^{i}\right\}^{\frac{1}{q}} \\
& +\left(\eta_{2}-z\right)^{\alpha+1}\left\{\sum_{i=1}^{n}\left(\frac{\gamma(\alpha+1,-s)-\Gamma(\alpha+1)}{(-s)^{\alpha} s}-\frac{1}{\alpha+1}\right)^{i}\right. \\
& \left.\left.-\sum_{i=1}^{n}\left((\gamma(\alpha+1, s)-\Gamma(\alpha+1)) s^{-\alpha-1} e^{s}-\frac{1}{\alpha+1}\right)^{i}\right\}^{\frac{1}{q}}\right] .
\end{aligned}
$$

Proof. Using Lemma 2 and power mean inequality, we have

$$
\begin{aligned}
& \left|\left(\frac{\left(z-\eta_{1}\right)^{\alpha}+\left(\eta_{2}-z\right)^{\alpha}}{\eta_{2}-\eta_{1}}\right) \varphi(z)-\frac{\Gamma(\alpha+1)}{\eta_{2}-\eta_{1}}\left\{J_{z^{-}}^{\alpha} \varphi\left(\eta_{1}\right)+J_{z^{+}}^{\alpha} \varphi\left(\eta_{2}\right)\right\}\right| \\
& \leq \frac{\left(z-\eta_{1}\right)^{\alpha+1}}{\eta_{2}-\eta_{1}} \int_{0}^{1} \chi^{\alpha}\left|\varphi^{\prime}\left(\chi z+(1-\chi) \eta_{1}\right)\right| d \chi+\frac{\left(\eta_{2}-z\right)^{\alpha+1}}{\eta_{2}-\eta_{1}} \int_{0}^{1} \chi^{\alpha}\left|\varphi^{\prime}\left(\chi z+(1-\chi) \eta_{2}\right)\right| d \chi \\
& \leq \frac{\left(z-\eta_{1}\right)^{\alpha+1}}{\eta_{2}-\eta_{1}}\left(\int_{0}^{1} \chi^{\alpha} d \chi\right)^{1-\frac{1}{q}}\left(\int_{0}^{1} \chi^{\alpha}\left|\varphi^{\prime}\left(\chi z+(1-\chi) \eta_{1}\right)\right|^{q} d \chi\right)^{\frac{1}{q}} \\
& +\frac{\left(\eta_{2}-z\right)^{\alpha+1}}{\eta_{2}-\eta_{1}}\left(\int_{0}^{1} \chi^{\alpha} d \chi\right)^{1-\frac{1}{q}}\left(\int_{0}^{1} \chi^{\alpha}\left|\varphi^{\prime}\left(\chi z+(1-\chi) \eta_{2}\right)\right|^{q} d \chi\right)^{\frac{1}{q}}
\end{aligned}
$$

Since, $\left|\varphi^{\prime}\right|^{q}$ is $n$-polynomial exponentially s-convexity and $\left|\varphi^{\prime}(z)\right| \leq K$, we obtain

$$
\begin{aligned}
& \int_{0}^{1} \chi^{\alpha}\left|\varphi^{\prime}\left(\chi z+(1-\chi) \eta_{1}\right)\right|^{q} d \chi \\
& =\int_{0}^{1} \chi^{\alpha}\left\{\frac{1}{n} \sum_{i=1}^{n}\left(e^{s \chi}-1\right)^{i}\left|\varphi^{\prime}(z)\right|^{q}+\frac{1}{n} \sum_{i=1}^{n}\left(e^{s(1-\chi)}-1\right)^{i}\left|\varphi^{\prime}\left(\eta_{1}\right)\right|^{q}\right\} d \chi \\
& \leq \frac{K^{q}}{n}\left\{\sum_{i=1}^{n}\left(\frac{\gamma(\alpha+1,-s)-\Gamma(\alpha+1)}{(-s)^{\alpha} s}-\frac{1}{\alpha+1}\right)^{i}\right. \\
& \left.-\sum_{i=1}^{n}\left((\gamma(\alpha+1, s)-\Gamma(\alpha+1)) s^{-\alpha-1} e^{s}-\frac{1}{\alpha+1}\right)^{i}\right\}
\end{aligned}
$$

Consequently, similar computation gives

$$
\begin{aligned}
& \int_{0}^{1} \chi^{\alpha}\left|\varphi^{\prime}\left(\chi z+(1-\chi) \eta_{2}\right)\right|^{q} d \chi \\
& =\int_{0}^{1} \chi^{\alpha}\left\{\frac{1}{n} \sum_{i=1}^{n}\left(e^{s \chi}-1\right)^{i}\left|\varphi^{\prime}(z)\right|^{q}+\frac{1}{n} \sum_{i=1}^{n}\left(e^{s(1-\chi)}-1\right)^{i}\left|\varphi^{\prime}\left(\eta_{2}\right)\right|^{q}\right\} d \chi \\
& \leq \frac{K^{q}}{n}\left\{\sum_{i=1}^{n}\left(\frac{\gamma(\alpha+1,-s)-\Gamma(\alpha+1)}{(-s)^{\alpha} s}-\frac{1}{\alpha+1}\right)^{i}\right. \\
& \left.-\sum_{i=1}^{n}\left((\gamma(\alpha+1, s)-\Gamma(\alpha+1)) s^{-\alpha-1} e^{s}-\frac{1}{\alpha+1}\right)^{i}\right\}
\end{aligned}
$$


By connecting (27) and (28) with (26), we obtain the desired result (25).

Corollary 11. If we assign $n=1$ in Theorem 4 , then

$$
\begin{aligned}
& \left|\left(\frac{\left(z-\eta_{1}\right)^{\alpha}+\left(\eta_{2}-z\right)^{\alpha}}{\eta_{2}-\eta_{1}}\right) \varphi(z)-\frac{\Gamma(\alpha+1)}{\eta_{2}-\eta_{1}}\left\{J_{z^{-}}^{\alpha} \varphi\left(\eta_{1}\right)+J_{z^{+}}^{\alpha} \varphi\left(\eta_{2}\right)\right\}\right| \\
& \leq \frac{K}{\left(\eta_{2}-\eta_{1}\right)}\left(\frac{1}{\alpha+1}\right)^{1-\frac{1}{q}} \\
& \times\left[\left(z-\eta_{1}\right)^{\alpha+1}\left\{\frac{\gamma(\alpha+1,-s)-\Gamma(\alpha+1)}{(-s)^{\alpha} s}-(\gamma(\alpha+1, s)-\Gamma(\alpha+1)) s^{-\alpha-1} e^{s}-\frac{2}{\alpha+1}\right\}^{\frac{1}{q}}\right. \\
& \left.+\left(\eta_{2}-z\right)^{\alpha+1}\left\{\frac{\gamma(\alpha+1,-s)-\Gamma(\alpha+1)}{(-s)^{\alpha} s}-(\gamma(\alpha+1, s)-\Gamma(\alpha+1)) s^{-\alpha-1} e^{s}-\frac{2}{\alpha+1}\right\}^{\frac{1}{q}}\right] .
\end{aligned}
$$

Corollary 12. If we assign $s=1$, in Theorem 4 , then

$$
\begin{aligned}
& \left|\left(\frac{\left(z-\eta_{1}\right)^{\alpha}+\left(\eta_{2}-z\right)^{\alpha}}{\eta_{2}-\eta_{1}}\right) \varphi(z)-\frac{\Gamma(\alpha+1)}{\eta_{2}-\eta_{1}}\left\{J_{z^{-}}^{\alpha} \varphi\left(\eta_{1}\right)+J_{z^{+}}^{\alpha} \varphi\left(\eta_{2}\right)\right\}\right| \\
& \leq \frac{K}{\sqrt[q]{n}\left(\eta_{2}-\eta_{1}\right)}\left(\frac{1}{\alpha+1}\right)^{1-\frac{1}{q}} \\
& \times\left[( z - \eta _ { 1 } ) ^ { \alpha + 1 } \left\{\sum_{i=1}^{n}\left(\frac{\gamma(\alpha+1,-1)-\Gamma(\alpha+1)}{(-1)^{\alpha}}-\frac{1}{\alpha+1}\right)^{i}\right.\right. \\
& \left.-\sum_{i=1}^{n}\left((\gamma(\alpha+1,1)-\Gamma(\alpha+1)) e-\frac{1}{\alpha+1}\right)^{i}\right\}^{\frac{1}{q}} \\
& +\left(\eta_{2}-z\right)^{\alpha+1}\left\{\sum_{i=1}^{n}\left(\frac{\gamma(\alpha+1,-1)-\Gamma(\alpha+1)}{(-1)^{\alpha}}-\frac{1}{\alpha+1}\right)^{i}\right. \\
& \left.\left.-\sum_{i=1}^{n}\left((\gamma(\alpha+1,1)-\Gamma(\alpha+1)) e-\frac{1}{\alpha+1}\right)^{i}\right\}^{\frac{1}{q}}\right] .
\end{aligned}
$$

Corollary 13. If we assign $\alpha=1$, in Theorem 4 , then

$$
\begin{aligned}
& \left|\varphi(z)-\frac{1}{\eta_{2}-\eta_{1}} \int_{\eta_{1}}^{\eta_{2}} \varphi(\chi) d \chi\right| \\
& \leq \frac{K}{\sqrt[q]{n}\left(\eta_{2}-\eta_{1}\right) 2^{1-\frac{1}{q}}}\left[\left(z-\eta_{1}\right)^{2}\left\{\sum_{i=1}^{n}\left(\frac{2+(2 s-2) e^{s}-s^{2}}{2 s^{2}}\right)^{i}+\sum_{i=1}^{n}\left(\frac{2 e^{s}-s^{2}-2 s-2}{2 s^{2}}\right)^{i}\right\}^{\frac{1}{q}}\right. \\
& \left.+\left(\eta_{2}-z\right)^{2}\left\{\sum_{i=1}^{n}\left(\frac{2+(2 s-2) e^{s}-s^{2}}{2 s^{2}}\right)^{i}+\sum_{i=1}^{n}\left(\frac{2 e^{s}-s^{2}-2 s-2}{2 s^{2}}\right)^{i}\right\}^{\frac{1}{q}}\right] .
\end{aligned}
$$

Corollary 14. If we assign $\alpha=1$ and $z=\eta_{1}$ in Theorem 4 , then

$$
\begin{aligned}
& \left|\varphi\left(\eta_{1}\right)-\frac{1}{\eta_{2}-\eta_{1}} \int_{\eta_{1}}^{\eta_{2}} \varphi(\chi) d \chi\right| \\
& \leq \frac{K}{\sqrt[q]{n}\left(\eta_{2}-\eta_{1}\right) 2^{1-\frac{1}{q}}}\left[\left(\eta_{2}-\eta_{1}\right)^{2}\left\{\sum_{i=1}^{n}\left(\frac{2+(2 s-2) e^{s}-s^{2}}{2 s^{2}}\right)^{i}+\sum_{i=1}^{n}\left(\frac{2 e^{s}-s^{2}-2 s-2}{2 s^{2}}\right)^{i}\right\}^{\frac{1}{q}}\right] .
\end{aligned}
$$


Corollary 15. If we assign $\alpha=1$ and $z=\eta_{2}$ in Theorem 4 , then

$$
\begin{aligned}
& \left|\varphi\left(\eta_{2}\right)-\frac{1}{\eta_{2}-\eta_{1}} \int_{\eta_{1}}^{\eta_{2}} \varphi(\chi) d \chi\right| \\
& \leq \frac{K}{\sqrt[q]{n}\left(\eta_{2}-\eta_{1}\right) 2^{1-\frac{1}{q}}}\left[\left(\eta_{2}-\eta_{1}\right)^{2}\left\{\sum_{i=1}^{n}\left(\frac{2+(2 s-2) e^{s}-s^{2}}{2 s^{2}}\right)^{i}+\sum_{i=1}^{n}\left(\frac{2 e^{s}-s^{2}-2 s-2}{2 s^{2}}\right)^{i}\right\}^{\frac{1}{q}}\right] .
\end{aligned}
$$

\section{Applications}

We recall the following special means for different positive real numbers $\eta_{1}, \eta_{2}$ and $\eta_{1}<\eta_{2}$ as follows:

1. The arithmetic mean:

$$
A\left(\eta_{1}, \eta_{2}\right)=\frac{\eta_{1}+\eta_{2}}{2}
$$

2. The Harmonic mean:

$$
H\left(\eta_{1}, \eta_{2}\right)=\frac{2 \eta_{1} \eta_{2}}{\eta_{1}+\eta_{2}}, \eta_{1}, \eta_{2}>0
$$

3. The logarithmic mean:

$$
L=L\left(\eta_{1}, \eta_{2}\right)=\frac{\eta_{2}-\eta_{1}}{\ln \eta_{2}-\ln \eta_{1}}, \quad \eta_{1} \neq \eta_{2}
$$

4. The generalized logarithmic mean:

$$
L_{r}\left(\eta_{1}, \eta_{2}\right)=\left[\frac{\eta_{2}^{r+1}-\eta_{1}^{r+1}}{(r+1)\left(\eta_{2}-\eta_{1}\right)}\right]^{\frac{1}{r}} ; r \in R \backslash\{-1,0\} .
$$

5. The Identric mean:

$$
I\left(\eta_{1}, \eta_{2}\right)=\left\{\begin{array}{cc}
\eta_{1} & \eta_{1}=\eta_{2} \\
\frac{1}{e}\left(\frac{\eta_{2}^{\eta_{2}}}{\eta_{1}^{\eta_{1}}}\right)^{\frac{1}{\eta_{2}-\eta_{1}}} & \eta_{1} \neq \eta_{2}
\end{array}\right\}
$$

Proposition 2. Let $0<\eta_{1}<\eta_{2}$. Then for some fixed $s \in[\ln 2.4,1)$, we obtain

$$
\begin{aligned}
& \left|\ln I\left(\eta_{1}, \eta_{2}\right)-\ln A\left(\eta_{1}, \eta_{2}\right)\right| \\
& \leq\left(\eta_{2}-\eta_{1}\right) \frac{K}{2 n}\left\{\sum_{i=1}^{n}\left(\frac{2+2(s-1) e^{s}-s^{2}}{2 s^{2}}\right)^{i}+\sum_{i=1}^{n}\left(\frac{2 e^{s}-s^{2}-2 s-2}{2 s^{2}}\right)^{i}\right\} .
\end{aligned}
$$

Proof. The assertion follows from Corollary 3 by letting $z=\frac{\eta_{1}+\eta_{2}}{2}$ and $\varphi(z)=-\ln z$.

Note: Estimation of " $K$ " for the above Proposition 2 is as follows:

$$
\varphi(z)=-\ln z \Longrightarrow\left|\varphi^{\prime}(z)\right|=\frac{1}{z} \leq K=\left|\varphi^{\prime}\left(\eta_{1}\right)\right|=\frac{1}{\eta_{1}}
$$

Proposition 3. Let $0<\eta_{1}<\eta_{2}$ and $q>1$. Then for some fixed $s \in[\ln 2.4,1)$, we obtain

$$
\left|H\left(\eta_{1}, \eta_{2}\right)-L^{-1}\left(\eta_{1}, \eta_{2}\right)\right| \leq 2^{\frac{1}{q}-1} \frac{K}{\sqrt[q]{n}}\left(\eta_{2}-\eta_{1}\right)\left(\frac{1}{p+1}\right)^{\frac{1}{p}}\left\{\sum_{i=1}^{n}\left(\frac{e^{s}-s-1}{s}\right)^{i}\right\}^{\frac{1}{q}} .
$$

Proof. The assertion follows from Corollary 8 by letting $z=\frac{\eta_{1}+\eta_{2}}{2}$ and $\varphi(z)=\frac{1}{z}$. 
Note: Estimation of " $K$ " for the above Proposition 3 is as follows:

$$
\varphi(z)=\frac{1}{z} \Longrightarrow\left|\varphi^{\prime}(z)\right|=\frac{1}{z^{2}} \leq K=\left|\varphi^{\prime}\left(\eta_{1}\right)\right|=\frac{1}{\eta_{1}^{2}}
$$

Proposition 4. Let $0<\eta_{1}<\eta_{2}$. Then for some fixed $s \in[\ln 2.4,1)$, we obtain

$$
\begin{aligned}
& \left|A^{l s}\left(\eta_{1}, \eta_{2}\right)-L_{l s}^{l s}\left(\eta_{1}, \eta_{2}\right)\right| \\
& \leq\left(\eta_{2}-\eta_{1}\right) \frac{K}{2 n}\left\{\sum_{i=1}^{n}\left(\frac{2+2(s-1) e^{s}-s^{2}}{2 s^{2}}\right)^{i}+\sum_{i=1}^{n}\left(\frac{2 e^{s}-s^{2}-2 s-2}{2 s^{2}}\right)^{i}\right\} .
\end{aligned}
$$

Proof. The assertion follows from Corollary 3 by letting $z=\frac{\eta_{1}+\eta_{2}}{2}$ and $\varphi(z)=z^{l s}$.

Note: Estimation of " $K$ " for the above Proposition 4 is as follows:

$$
\varphi(z)=z^{l s} \Longrightarrow\left|\varphi^{\prime}(z)\right|=l s(z)^{l s-1} \leq K=\left|\varphi^{\prime}\left(\eta_{2}\right)\right|=l s\left(\eta_{2}\right)^{l s-1}
$$

Proposition 5. Let $0<\eta_{1}<\eta_{2}$. Then for some fixed $s \in[\ln 2.4,1)$, we obtain

$$
\begin{aligned}
& \left|A^{l s}\left(\eta_{1}, \eta_{2}\right)-L_{l s}^{l s}\left(\eta_{1}, \eta_{2}\right)\right| \\
& \leq 2^{\frac{1}{q}-1} \frac{K}{\sqrt[q]{n}}\left(\eta_{2}-\eta_{1}\right)\left(\frac{1}{p+1}\right)^{\frac{1}{p}}\left\{\sum_{i=1}^{n}\left(\frac{e^{s}-s-1}{s}\right)^{i}\right\}^{\frac{1}{q}} .
\end{aligned}
$$

Proof. The assertion follows from Corollary 8 by letting $z=\frac{\eta_{1}+\eta_{2}}{2}$ and $\varphi(z)=z^{l s}$.

Note: Similarly, one can estimate the value of " $K$ " as estimated in the above propositions (see Propositions 2-4).

Proposition 6. Let $0<\eta_{1}<\eta_{2}$. Then for some fixed $s \in[\ln 2.4,1)$, we obtain

$$
\begin{aligned}
& \left|G^{S}(\alpha, \beta)-L\left(\alpha^{s}, \beta^{s}\right)\right| \\
& \leq\left(\eta_{2}-\eta_{1}\right) \frac{K}{2 n}\left\{\sum_{i=1}^{n}\left(\frac{2+2(s-1) e^{s}-s^{2}}{2 s^{2}}\right)^{i}+\sum_{i=1}^{n}\left(\frac{2 e^{s}-s^{2}-2 s-2}{2 s^{2}}\right)^{i}\right\} .
\end{aligned}
$$

Proof. The assertion follows from Corollary 3 by letting $z=\frac{\eta_{1}+\eta_{2}}{2}, \varphi(z)=e^{s z}, z>0$ and $\alpha=e^{\eta_{1}}, \beta=e^{\eta_{2}}$.

Note: Similarly, one can estimate the value of " $K$ " as estimated in the above propositions (see Propositions 2-4).

\section{Midpoint Formula}

Since in [36], suppose $d$ is the division $\eta_{1}=x_{0}<x_{1}<x_{2}<\ldots<x_{n}=\eta_{2}$ of the interval $\left[\eta_{1}, \eta_{2}\right]$ and consider the quadrature formula

$$
\int_{\eta_{1}}^{\eta_{2}} \varphi(\chi) d \chi=\mathrm{T}(\varphi, d)+E(\varphi, d)
$$

where $\mathrm{T}(\varphi, d)=\sum_{j=1}^{n-1} \varphi\left(\frac{x_{j}+x_{j+1}}{2}\right) h_{j}$, is the midpoint version and $E(\varphi, d)$ denotes the approximation error and $h_{j}=x_{j+1}-x_{j}$, for $j=0,1,2, \ldots, n-1$. 
Proposition 7. Suppose a mapping $\varphi: I \subset[0, \infty) \rightarrow \mathbb{R}$ is differentiable on $I^{0}$ such that $\varphi^{\prime} \in L\left[\eta_{1}, \eta_{2}\right]$, where $\eta_{1}, \eta_{2} \in$ I with $\eta_{2}>\eta_{1}$. If $\left|\varphi^{\prime}\right|$ is $n$-polynomial exponentially s-convex on $\left[\eta_{1}, \eta_{2}\right]$, then for every division $d$ of $\left[\eta_{1}, \eta_{2}\right]$, the midpoint error satisfy

$$
|E(\varphi, d)| \leq \frac{K}{2 n} \sum_{j=0}^{n-1} h_{j}^{2}\left\{\sum_{i=1}^{n}\left(\frac{2+2(s-1) e^{s}-s^{2}}{2 s^{2}}\right)^{i}+\sum_{i=1}^{n}\left(\frac{2 e^{s}-s^{2}-2 s-2}{2 s^{2}}\right)^{i}\right\} .
$$

Proof. Since applying Corollary 3 with $n$-polynomial exponentially $s$-convexity and $z=\frac{\eta_{1}+\eta_{2}}{2}$ on the subinterval $\left[x_{i}, x_{j+1}\right]$

$$
\begin{aligned}
& \left|h_{j} \varphi\left(\frac{x_{j}+x_{j+1}}{2}\right)-\int_{x_{j}}^{x_{j+1}} \varphi(x) d x\right| \leq \frac{h_{j}^{2} K}{2 n} \\
& \times\left\{\sum_{i=1}^{n}\left(\frac{2+2(s-1) e^{s}-s^{2}}{2 s^{2}}\right)^{i}+\sum_{i=1}^{n}\left(\frac{2 e^{s}-s^{2}-2 s-2}{2 s^{2}}\right)^{i}\right\} .
\end{aligned}
$$

Summing over $j$ from 0 to $\mathrm{n}-1$ and taking into account that $\left|\varphi^{\prime}\right|$ is $n$-polynomial exponentially $s$-convex, we obtain, by the triangle inequality

$$
\begin{aligned}
& \left|\int_{\eta_{1}}^{\eta_{2}} \varphi(\chi) d \chi-\mathrm{T}(\varphi, d)\right| \\
& \leq\left|\sum_{j=0}^{n-1}\left\{\int_{x_{j}}^{x_{j+1}} \varphi(x) d x-\varphi\left(\frac{x_{j}+x_{j+1}}{2}\right) h_{j}\right\}\right| \\
& \leq \sum_{j=0}^{n-1}\left|\left\{\int_{x_{j}}^{x_{j+1}} \varphi(x) d x-\varphi\left(\frac{x_{j}+x_{j+1}}{2}\right) h_{j}\right\}\right| \\
& \leq \frac{K}{2 n} \sum_{j=0}^{n-1} h_{j}^{2} \times\left\{\sum_{i=1}^{n}\left(\frac{2+2(s-1) e^{s}-s^{2}}{2 s^{2}}\right)^{i}+\sum_{i=1}^{n}\left(\frac{2 e^{s}-s^{2}-2 s-2}{2 s^{2}}\right)^{i}\right\} .
\end{aligned}
$$

which completes the proof.

Note: Similarly, one can estimate the value of " $K$ " as estimated in the above propositions (see Propositions 2-4).

Proposition 8. Suppose a mapping $\varphi: I \subset[0, \infty) \rightarrow \mathbb{R}$ is differentiable on $I^{0}$ such that $\varphi^{\prime} \in L\left[\eta_{1}, \eta_{2}\right]$, where $\eta_{1}, \eta_{2} \in I$ with $\eta_{2}>\eta_{1}, q>1$, for $s \in[\ln 2.4,1)$ in (29), for every division $d$ of $\left[\eta_{1}, \eta_{2}\right]$. If $\left|\varphi^{\prime}\right|^{q}$ is $n$-polynomial exponentially s-convex on $\left[\eta_{1}, \eta_{2}\right]$, then the midpoint error satisfy

$$
|E(\varphi, d)| \leq 2^{\frac{1}{q}-1} \frac{K}{\sqrt[q]{n}} \sum_{j=0}^{n-1} h_{j}^{2} \times\left(\frac{1}{p+1}\right)^{\frac{1}{p}}\left\{\sum_{i=1}^{n}\left(\frac{e^{s}-s-1}{s}\right)^{i}\right\}^{\frac{1}{q}} .
$$

Proof. By applying the same technique as in proposition (7) but using the Corollary 8 with $z=\frac{\eta_{1}+\eta_{2}}{2}$.

Note: Similarly, one can estimate the value of " $K$ " as estimated in the above propositions (see Propositions 2-4).

\section{Conclusions}

In this article, we have taken into consideration a critical extension of convexity that is referred to as $n$-polynomial exponentially s-convex functions and acquired a new Hermite-Hadamard-type inequality and some novel refinements of Ostrowski-type inequalities. We also presented some applications of our established results to special means of two positive real numbers and midpoint formula. In the future, new inequalities for 
other $n$-polynomial convex functions can be obtained by using the techniques used in this article.

Author Contributions: Conceptualization, S.K.S., M.T., H.A. (Hijaz Ahmad), J.N., H.A. (Hassen Aydi); methodology, S.K.S., M.T., H.A. (Hijaz Ahmad), J.N., H.A. (Hassen Aydi); validation, S.K.S., M.T., H.A. (Hijaz Ahmad), J.N., H.A. (Hassen Aydi), A.M.; investigation, S.K.S., M.T., H.A. (Hijaz Ahmad), J.N., H.A. (Hassen Aydi); writing— original draft preparation, S.K.S., M.T.; writing-review and editing, S.K.S., M.T., H.A. (Hijaz Ahmad), J.N., H.A. (Hassen Aydi), A.M.; supervision, S.K.S., M.T., H.A. (Hassen Aydi). All authors have read and agreed to the final version of the manuscript.

Funding: Research group Nonlinear Analysis Methods in Applied Mathematics (NAMAM) group number RG-DES-2017-01-17, Prince Sultan University.

Institutional Review Board Statement: Not applicable.

Informed Consent Statement: Not applicable.

Data Availability Statement: No data and materials were used to support this study.

Acknowledgments: The last author would like to thank Prince Sultan University for funding this work through research group Nonlinear Analysis Methods in Applied Mathematics (NAMAM) group number RG-DES-2017-01-17.

Conflicts of Interest: The authors declare that they have no competing interests.

\section{References}

1. Mohammed, P.O.; Brevik, I. A new version of the Hermite-Hadamard inequality for Riemann-Liouville fractional integrals. Symmetry 2020, 12, 610. [CrossRef]

2. Mohammed, P.O.; Sarikaya, M.Z.; Baleanu, D. On the generalized Hermite-Hadamard inequalities via the tempered fractional integrals. Symmetry 2020, 12, 595. [CrossRef]

3. Mohammed, P.O.; Abdeljawad, T. Modification of certain fractional integral inequalities for convex functions. Adv. Differ. Equ. 2020, 2020, 1-22. [CrossRef]

4. Vivas-Cortez, M.; Kashuri, A.; Hernández, J.E.H. Trapezium-type inequalities for Raina's fractional integrals operator using generalized convex functions. Symmetry 2020, 12, 1034. [CrossRef]

5. Butt, S.I.; Nadeem, M.; Qaisar, S.; Akdemir, A.O.; Abdeljawad, T. Hermite-Jensen-Mercer type inequalities for conformable integrals and related results. Adv. Differ. Equ. 2020, 1, 1-24. [CrossRef]

6. Sarikaya, M.Z.; Set, E.; Yaldiz, H.; Başak, N. Hermite-Hadamard inequalities for fractional integrals and related fractional inequalities. Math. Comput. Model. 2013, 57, 2403-2407. [CrossRef]

7. Mohammed, P.O.; Aydi, H.; Kashuri, A.; Hamed, Y.S.; Abualnaja, K.M. Midpoint inequalities in fractional calculus defined using positive weighted symmetry function kernels. Symmetry 2021, 13, 550. [CrossRef]

8. Kilbas, A.A.; Srivastava, H.M.; Trujillo, J.J. Theory and Applications of Fractional Differential Equations; Elsevier: Amsterdam, The Netherlands, 2006.

9. Xi, B.Y.; Qi, F. Some integral inequalities of Hermite-Hadamard type for convex functions with applications to means. J. Funct. Spaces Appl. 2012, 2012, 980438. [CrossRef]

10. Özcan, S.; İşcan, İ. Some new Hermite-Hadamard type integral inequalities for the $s$-convex functions and theirs applications. J. Inequal. Appl. 2019, 201,1-14.

11. Mehren, K.; Agarwal, P. New Hermite-Hadamard type integral inequalities for the convex functions and theirs applications. $J$. Comp. Appl. Math. 2019, 350, 274-285. [CrossRef]

12. Butt, S.I.; Kashuri, A.; Tariq, M.; Nasir, J.; Aslam, A.; Geo, W. n-polynomial exponential-type $p$-convex function with some related inequalities and their applications. Heliyon 2020, 6, e05420. [CrossRef] [PubMed]

13. Zhang, K.S.; Wan, J.P. p-convex functions and their applications. Pure Appl. Math. 2017, 23, 130-133

14. Butt, S.I.; Tariq, M.; Aslam, A.; Ahmad, H.; Nofel, T.A. Hermite-Hadamard type inequalities via generalized harmonic exponential convexity. J. Funct. Spaces 2021. [CrossRef]

15. Butt, S.I.; Kashuri, A.; Tariq, M.; Nasir, J.; Aslam, A.; Geo, W. Hermite-Hadamard type inequalities via n-polynomial exponentialtype convexity and their applications. Adv. Differ. Equ. 2020, 508, 1-25. [CrossRef]

16. Dragomir, S.S. Ostrowski type inequalities for Riemann-Liouville fractional integrals of absolutely continuous functions in terms of norms. RGMIA Res. Rep. Collect. 2017, 20, 49.

17. Mitrinovic, D.S.; Pecaric, J.; Fink, A.M. Inequalities Involving Functions and Their Integrals and Derivatives; Springer Science and Business Media: Berlin/Heidelberg, Germany, 1991; Volume 53, 603p

18. Alomari, M.; Darus, M. Some Ostrowski type inequalities for quasi-convex functions with applications to special means. RGMIA Res. Rep. Coll 2010, 13696936, 1-9 
19. Cerone, P.; Dragomir, S.S. Ostrowski type inequalities for functions whose derivatives satisfy certain convexity assumptions. Demonstr. Math. 2004, 37, 299-308. [CrossRef]

20. Alomari, M.; Darus, M.; Dragomir, S.S.; Cerone, P. Ostrowski type inequalities for functions whose derivatives are s-convex in the second sense. Appl. Math. Lett. 2010, 23, 1071-1076. [CrossRef]

21. Dragomir, S.S. On the Ostrowski's integral inequality for mappings with bounded variation and applications. Math. Ineq. Appl. 1998, 1, 59-66. [CrossRef]

22. Set, E.; Sarikaya, M.Z.; Özdemir, M.E. Some Ostrowski's Type Inequalities for Functions whose Second Derivatives are s-Convex in the Second Sense and Applications. arXiv 2010, arXiv:1006.2488.

23. Pachpatte, B.G. On an inequality of Ostrowski type in three independent variables. J. Math. Anal. Appl. 2000, $249,583-591$. [CrossRef]

24. Antczak, T. On (p,r)-invex set and functions. J. Math. Anal. Appl. 2001, 263, 355-379. [CrossRef]

25. Dragomir, S.S.; Gomm, I. Some Hermite-Hadamard's Inequality functions whose exponentials are convex. Babes Bolyani Math. 2005, 60, 527-534.

26. Awan, M.U.; Noor, M.A.; Noor, K.I. Hermite-Hadamard type inequalities for exponentially convex function. Appl. Math. Inf. Sci. 2018, 12, 405-409. [CrossRef]

27. Kadakal, M.; İşcan, İ. Exponential type convexity and some related inequalities. J. Inequal. Appl. 2020, 2020, 1-9. [CrossRef]

28. Niculescu, C.P.; Persson, L.E. Convex Functions and Their Applications; Springer: New York, NY, USA, 2006.

29. Dragomir, S.S.; Fitzpatrik, S. The Hadamard inequality for $s$-convex functions in the second sense. Demonstr. Math. 1999, 32, 687-696. [CrossRef]

30. Set, E.; Özdemir, M.E.; Sarikaya, M.Z. New inequalities of Ostrowski's type for s-convex functions in the second sense with applications. arXiv 2010, arXiv:1005.0702.

31. Breckner, W.W. Stetigkeitsaussagen für eine Klasse verallgemeinerter konvexer funktionen in topologischen linearen Raumen. Pupl. Inst. Math. 1978, 23, 13-20.

32. Hudzik, H.; Maligranda, L. Some remarks on s-convex functions. Aequ. Math. 1994, 48, 100-111. [CrossRef]

33. Toplu, T.; Kadakal, M.; İşcan, İ. On n-polynomial convexity and some relatd inequalities. AIMS 2020, 5, 1304-1318. [CrossRef]

34. Noor, M.A.; Noor, K.I.; Awan, M.U. Fractional Ostrowski inequalities for s-Godunova-Levin functions. Int. J. Anal. Appl. 2014, 5, 167-173.

35. Set, E. New inequalities of Ostrowski type for mappings whose derivatives are s-convex in the second sense via fractional integrals. Comput. Math. Appl. 2012, 63, 1147-1154. [CrossRef]

36. Kirmaci, U.S.; Özdemir, M.E. On some inequalities for differentiable mappings and applications to special means of real numbers and to midpoint formula. Appl. Math. Comput. 2004, 153, 361-368. [CrossRef] 